\title{
Alain Prignet \\ Conditions aux limites non homogènes pour des problèmes elliptiques avec second membre mesure
}

Annales de la faculté des sciences de Toulouse $6^{e}$ série, tome $6, \mathrm{n}^{\circ} 2$ (1997), p. 297-318

<http://www.numdam.org/item?id=AFST_1997_6_6_2_297_0>

(C) Université Paul Sabatier, 1997, tous droits réservés.

L'accès aux archives de la revue «Annales de la faculté des sciences de Toulouse » (http://picard.ups-tlse.fr/ annales/) implique l'accord avec les conditions générales d'utilisation (http://www.numdam.org/conditions). Toute utilisation commerciale ou impression systématique est constitutive d'une infraction pénale. Toute copie ou impression de ce fichier doit contenir la présente mention de copyright.

\section{Numdam}

Article numérisé dans le cadre du programme Numérisation de documents anciens mathématiques http://www.numdam.org/ 


\title{
Conditions aux limites non homogènes pour des problèmes elliptiques avec second membre mesure ${ }^{(*)}$
}

\author{
Alain Prignet ${ }^{(1)}$
}

RÉsuMÉ. - Pour $\Omega$ ouvert borné régulier de $\mathbb{R}^{N}$, on étudie le problème elliptique

$$
-\operatorname{div}(A(x, u, \nabla u))=f \quad \operatorname{dans} \mathcal{D}^{\prime}(\Omega)
$$

où $f$ est une mesure de Radon, et nous montrons l'existence d'une solution vérifiant des conditions aux limites non homogènes de Neumann, Fourier et Dirichlet.

Abstract. - For $\Omega$ a smooth open bounded set of $\mathbb{R}^{N}$, we study the elliptic problem

$$
-\operatorname{div}(A(x, u, \nabla u))=f \quad \text { in } \mathcal{D}^{\prime}(\Omega)
$$

where $f$ is a Radon measure, and we show existence of a solution verifying non homogenous boundary conditions of Neumann, Fourier and Dirichlet.

\section{Introduction}

Pour $\Omega$ ouvert borné régulier de $\mathbb{R}^{N}$ avec $N \geq 2$, on étudie le problème elliptique

$$
-\operatorname{div}(A(x, u, \nabla u))=f \quad \operatorname{dans} \mathcal{D}^{\prime}(\Omega)
$$

avec $f \in M(\bar{\Omega})$, l'espace des mesures de Radon $\left(M(\bar{\Omega})=(C(\bar{\Omega}))^{\prime}\right.$ est le dual de l'espace des fonctions continues sur $\bar{\Omega}$ muni de sa norme habituelle) et avec des conditions aux limites non homogènes de Neumann, Fourier et Dirichlet.

(*) Reçu le 14 mars 1995

(1) UMPA ENS-Lyon, 46 allée d'Italie, F-69364 Lyon Cedex 07 (France) 
Ce problème a été étudié dans le cas de conditions homogènes de Dirichlet par Boccardo et Gallouët dans [2] et [3], où il est montré l'existence d'une solution. Cependant il n'y a pas unicité comme le montre le contre-exemple de Serrin ([11], [10]). Pour y remédier des solutions entropiques [1] et renormalisées ([8], [9]) ont été introduites dans le cas où $f \in L^{1}(\Omega)$. Nous ne montrerons donc ici que l'existence d'une solution pour un second membre mesure.

Le principe de la démonstration consiste à construire les solutions de problèmes approchés puis à considérer la limite de ces solutions. Pour cela, on régularise le second membre et les conditions aux limites. Nous nous appuierons sur les démonstrations de [4] et [3] pour les étapes 1 et sur celle de [1], [6] et [5] pour les étapes 2.

Donnons les hypothèses sur $A$, opérateur de Leray-Lions, nous les appellerons (H) par la suite : $A$ est une fonction de Carathéodory, c'est-à-dire que :

- $A(x, s, \xi): \Omega \times \mathbb{R} \times \mathbb{R}^{N} \rightarrow \mathbb{R}^{N}$ est mesurable en $x \in \Omega$ pour tout $s \in \mathbb{R}$ et $\xi \in \mathbb{R}^{N}$ et continue en $\xi \in \mathbb{R}^{N}$ et $s \in \mathbb{R}$ pour presque tout $x \in \Omega$.

Nous noterons

$$
A(x, u, \nabla u)=A(x, u(x), \nabla u(x))
$$

$A$ vérifie aussi des conditions de coercivité, monotonie et croissance $:$ il existe $p$ vérifiant $2-1 / N<p \leq N$ et :

- il existe $\alpha>0$ tel que pour tout $s$ et $\xi$ et presque tout $x$, on ait

$$
A(x, s, \xi) \xi \geq \alpha|\xi|^{p}
$$

- pour tout $s, \xi$ et $\eta$ et presque tout $x$, on a

$$
(A(x, s, \xi)-A(x, s, \eta))(\xi-\eta)>0 \text { pour } \xi \neq \eta ;
$$

- il existe $b(x) \in L^{p^{\prime}}(\Omega), p^{\prime}=p /(p-1)$, et $\beta>0$ tels que pour tout $s$ et $\xi$ et presque tout $x$, on ait

$$
|A(x, s, \xi)| \leq \beta\left(b(x)+|s|^{p-1}+|\xi|^{p-1}\right) .
$$

Ces hypothèses sont classiques pour l'étude des opérateurs non linéaires sous forme divergentielle (voir Leray-Lions [7]). 
Pour $k>0$, nous utiliserons la fonction "tronquante" $T_{k}: \mathbb{R} \rightarrow \mathbb{R}$ telle que $T_{k}(x)=\min (k, \max (x,-k))$. Cette fonction étant lipschitzienne, si $u \in W^{1, p}(\Omega)$, on a $T_{k}(u) \in W^{1, p}(\Omega) \cap L^{\infty}(\Omega)$, cette dernière fonction pourra donc servir de fonction test.

Nous noterons $C$ toute constante indépendante de $n$, indice des suites qui interviendront par la suite.

\section{Conditions aux limites de Neumann}

Le problème de Neumann consiste à chercher $u$ telle que

$$
\begin{aligned}
-\operatorname{div}(A(x, u, \nabla u))=f & \operatorname{dans} \Omega \\
A(x, u, \nabla u) \cdot n=g & \text { sur } \partial \Omega
\end{aligned}
$$

avec $f \in M(\bar{\Omega})$ et $g \in M(\partial \Omega)$, où $n$ désigne, ici, la normale à $\partial \Omega$. On résoudra (2.1) au sens faible suivant : on cherche

$$
u \in \bigcap_{q<(p-1) N /(N-1)} W^{1, q}(\Omega)
$$

tel que

$$
\forall \varphi \in \bigcup_{r>N} W^{1, r}(\Omega), \quad \int_{\Omega} A(x, u, \nabla u) \nabla \varphi=\int_{\partial \Omega} \varphi \mathrm{d} g+\int_{\Omega} \varphi \mathrm{d} f .
$$

où l'on note encore $\varphi$, la trace de $\varphi$ sur $\partial \Omega$, ce que nous ferons désormais.

Bien entendu, si ce problème admet une solution, le choix $\varphi \equiv 1$ impose

$$
\int_{\partial \Omega} \mathrm{d} g+\int_{\Omega} \mathrm{d} f=0
$$

ce que nous allons supposer. Ce problème admet alors, en particulier, une infinité de solutions : plus précisément, il existe une solution de moyenne $C$, pour toute constante $C$. Aussi cherche-t-on les solutions à moyenne donnée.

THÉORÈme .- Soient $f \in M(\bar{\Omega})$ (ne chargeant pas le bord), $g \in$ $M(\partial \Omega)$, vérifiant (2.2), $\Omega$ un ouvert borné régulier, A vérifiant les hypothèses (H) et soit $\tilde{u} \in \mathbb{R}$, alors le problème (2.1) admet une solution de moyenne $\tilde{u}$. 


\section{Alain Prignet}

Démonstration. - Soient

$$
\left(f_{n}\right) \in W^{-1, p^{\prime}}(\Omega) \cap L^{1}(\Omega) \quad \text { et } \quad\left(g_{n}\right) \in\left(W^{1-1 / p, p}(\partial \Omega)\right)^{\prime} \cap L^{1}(\partial \Omega)
$$

tels que

$$
\left\|f_{n}\right\|_{L^{1}} \leq\|f\|_{M(\bar{\Omega})}, \quad\left\|g_{n}\right\|_{L^{1}} \leq\|g\|_{M(\partial \Omega)}
$$

et $f_{n} \rightarrow f$ dans $M(\bar{\Omega}) *$-faible et $g_{n} \rightarrow g$ dans $M(\partial \Omega) *$-faible tels que

$$
\int_{\partial \Omega} g_{n}+\int_{\Omega} f_{n}=0
$$

Soit $u_{n} \in W^{1, p}(\Omega)$ une solution de (2.1), avec $f=f_{n}$ et $g=g_{n}$, telle que

$$
\frac{1}{|\Omega|} \int_{\Omega} u_{n}=\tilde{u} \in \mathbb{R}
$$

(son existence est donnée par Leray et Lions [7]); elle vérifie

$$
\forall \varphi \in W^{1, p}(\Omega) \cap L^{\infty}(\Omega), \quad \int_{\Omega} A\left(x, u_{n}, \nabla u_{n}\right) \nabla \varphi=\int_{\partial \Omega} \varphi g_{n}+\int_{\Omega} \varphi f_{n} .
$$

\section{Étape 1}

Montrons que $u_{n}$ est borné dans $W^{1, q}(\Omega)$ pour $q<(p-1) N /(N-1)$, ce qui donnera l'existence de $u \in W^{1, q}(\Omega)$ tel qu'une sous-suite de $u_{n}$ converge vers $u$ dans $W^{1, q}(\Omega)$ faible.

Pour $m>0$ donné, soit

$$
\psi_{m}(x)= \begin{cases}m \int_{0}^{x} \mathrm{~d} t /(t+1)^{m+1} & \text { pour } x \geq 0 \\ -\psi_{m}(-x) & \text { pour } x \leq 0,\end{cases}
$$

on a $\left|\psi_{m}\right| \leq 1,\left|\psi_{m}^{\prime}\right| \leq m$ et $\psi_{m}$ continue. Alors $\psi_{m}\left(u_{n}\right) \in W^{1, p}(\Omega) \cap L^{\infty}(\Omega)$, il est donc possible de choisir $\varphi=\psi_{m}\left(u_{n}\right)$ dans (2.3) qui devient

$$
\int_{\Omega} A\left(x, u_{n}, \nabla u_{n}\right) \nabla \psi_{m}\left(u_{n}\right)=\int_{\partial \Omega} \psi_{m}\left(u_{n}\right) g_{n}+\int_{\Omega} \psi_{m}\left(u_{n}\right) f_{n}
$$

or $\nabla \psi_{m}\left(u_{n}\right)=\psi_{m}^{\prime}\left(u_{n}\right) \nabla u_{n}$ donc par coercitivité

$$
\alpha \int_{\Omega}\left|\nabla u_{n}\right|^{p} \psi_{m}^{\prime}\left(u_{n}\right) \leq\left\|f_{n}\right\|_{L^{1}}+\left\|g_{n}\right\|_{L^{1}}
$$


Conditions aux limites non homogènes pour des problèmes elliptiques

et $\psi_{m}^{\prime}(x)=m /(|x|+1)^{m+1}$ donc

$$
\alpha \int_{\Omega} m \frac{\left|\nabla u_{n}\right|^{p}}{\left(\left|u_{n}\right|+1\right)^{m+1}} \leq C
$$

grâce à l'inégalité de Hölder, nous obtenons pour $q<p$ :

$$
\int_{\Omega}\left|\nabla u_{n}\right|^{q} \leq\left(\int_{\Omega} \frac{\left|\nabla u_{n}\right|^{p}}{\left(\left|u_{n}\right|+1\right)^{m+1}}\right)^{q / p}\left(\int_{\Omega}\left(\left|u_{n}\right|+1\right)^{(m+1) q /(p-q)}\right)^{(p-q) / p} .
$$

Choisissons alors $m>0$ tel que $(m+1) q /(p-q)<q^{*}, 1 / q^{*}=1 / q-1 / N$, ce qui est possible pour $q<(p-1) N /(N-1)$, alors

$$
\left(\left|u_{n}\right|+1\right)^{(m+1) q /(p-q)} \leq \varepsilon\left|u_{n}\right|^{q^{*}}+C(\varepsilon)
$$

pour $\varepsilon>0$ petit, où $C(\varepsilon)$ dépend de $\varepsilon$, on obtient donc

$$
\int_{\Omega}\left|\nabla u_{n}\right|^{q} \leq C \varepsilon^{(p-q) / p}\left(\int_{\Omega}\left|u_{n}\right|^{q^{*}}\right)^{(p-q) / p}+C .
$$

Or la moyenne de $u_{n}$ est $\tilde{u}$, donc l'inégalité de Poincaré-Sobolev nous donne

$$
\left\|u_{n}-\tilde{u}\right\|_{L q^{*}} \leq C\left\|\nabla u_{n}\right\|_{L^{q}}
$$

soit

$$
\left\|u_{n}\right\|_{L^{q^{*}}} \leq C\left\|\nabla u_{n}\right\|_{L^{q}}+|\widetilde{u}|(\operatorname{mes}(\Omega))^{1 / q^{*}}
$$

d'où grâce à (2.4)

$$
\begin{aligned}
\left(\int_{\Omega}\left|u_{n}\right|^{q^{*}}\right)^{1 / q^{*}} & \leq C \varepsilon^{(p-q) / p q}\left(\int_{\Omega}\left|u_{n}\right|^{q^{*}}\right)^{(p-q) / p q}+C \\
& \leq \frac{1}{2}\left(\int_{\Omega}\left|u_{n}\right|^{q^{*}}\right)^{(p-q) / p q}+C
\end{aligned}
$$

pour $\varepsilon$ assez petit. Or $(p-q) / p q \leq 1 / q^{*}$ pour $p \leq N$ donc $\left\|u_{n}\right\|_{L^{q^{*}}} \leq C$ et donc, grâce à (2.4), $\int_{\Omega}\left|\nabla u_{n}\right|^{q} \leq C$. Ainsi $u_{n}$ est bornée dans $W^{1, q}(\Omega)$ pour tout $q<(p-1) N /(N-1)$; il est donc possible d'extraire de $u_{n}$ une sous-suite convergeant faiblement dans $W^{1, q}(\Omega)$. 


\section{Alain Prignet}

Étape 2

Afin de passer à la limite dans $A\left(x, u_{n}, \nabla u_{n}\right)$, la convergence presque partout de $u_{n}$ et $\nabla u_{n}$ est nécessaire. Celle de $u_{n}$ s'obtient par extraction d'une sous-suite car $u_{n}$ converge dans $L^{q}(\Omega)$ (théorème de Rellich), mais il faut montrer le résultat pour $\nabla u_{n}$.

Pour cela, montrons que $\nabla u_{n}$ tend vers $\nabla u$ en mesure, ce qui entraînera $\nabla u_{n} \rightarrow \nabla u$ presque partout, pour une sous-suite. Cela consiste à montrer que

$\forall \delta, \forall \varepsilon, \exists n_{0}$ tel que $\forall n \geq n_{0}, \quad \operatorname{mes}\left\{x,\left|\left(\nabla u_{n}-\nabla u\right)(x)\right| \geq \delta\right\} \leq \varepsilon ;$

remarquons que pour $k>0$ et $\eta>0$, on a

$$
\begin{aligned}
& \left\{\left|\left(\nabla u_{n}-\nabla u\right)(x)\right| \geq \delta\right\} \subset \\
& \subset\left\{\left|u_{n}\right| \geq k\right\} \cup\{|u| \geq k\} \cup\left\{\left|\nabla u_{n}\right| \geq k\right\} \cup \\
& \quad \cup\{|\nabla u| \geq k\} \cup \cup\left\{\left|u_{n}-u\right| \geq \eta\right\} \cup \\
& \quad \cup\left\{\left|\left(\nabla u_{n}-\nabla u\right)(x)\right| \geq \delta,\left|u_{n}\right| \leq k,|u| \leq k,\left|\nabla u_{n}\right| \leq k,\right. \\
& \left.\quad|\nabla u| \leq k,\left|u_{n}-u\right| \leq \eta\right\} ;
\end{aligned}
$$

nous appellerons $A_{1}$ à $A_{6}$ les six ensembles du membre de droite. On pourra remarquer, dans la suite de la démonstration, que seule la majoration de la mesure de $A_{6}$ fait intervenir (2.3), l'équation dont $u_{n}$ est solution.

Majorons $\operatorname{mes}\left(A_{1}\right)$, nous avons

$$
\int_{\Omega}\left|u_{n}\right| \geq \int_{A_{1}}\left|u_{n}\right| \geq k \operatorname{mes}\left(A_{1}\right)
$$

donc

$$
\operatorname{mes}\left(A_{1}\right) \leq \frac{1}{k} \int_{\Omega}\left|u_{n}\right| \leq \frac{C}{k} \leq \varepsilon
$$

pour $k$ assez grand, puisque $u_{n}$ est borné dans $W^{1, q}(\Omega)$ pour $q<(p-$ 1) $N /(N-1)$ et donc dans $L^{1}(\Omega)$. De même, $\nabla u_{n}$ est borné dans $L^{1}(\Omega)$ et $u \in W^{1, q}(\Omega) \subset W^{1,1}(\Omega)$, donc les mesures de $A_{2}, A_{3}$ et $A_{4}$ sont majorées de la même façon. Fixons $k$ tel que chacune des mesures soit plus petite que $\varepsilon$. 
Conditions aux limites non homogènes pour des problèmes elliptiques

Majorons maintenant mes $\left(A_{5}\right)$, nous avons

$$
\int_{\Omega}\left|\left(u_{n}-u\right)\right| \geq \int_{A_{5}}\left|\left(u_{n}-u\right)\right| \geq \eta \operatorname{mes}\left(A_{5}\right)
$$

or $u_{n}$ converge vers $u$ dans $L^{1}(\Omega)$ grâce au théorème de Rellich donc, pour $\eta$ donné, il existe $n_{1}$ tel que pour $n \geq n_{1}$ on ait

$$
\operatorname{mes}\left(A_{5}\right) \leq \varepsilon .
$$

Il reste donc à majorer mes $\left(A_{6}\right)$ et à choisir $\eta$. Grâce à la monotonie de $A$, nous avons

$$
\left(A\left(x, s, \xi_{1}\right)-A\left(x, s, \xi_{2}\right)\right)\left(\xi_{1}-\xi_{2}\right)>0 \quad \text { pour } \xi_{1}-\xi_{2} \neq 0 ;
$$

or l'ensemble des $\left(s, \xi_{1}, \xi_{2}\right)$ tels que $|s| \leq k,\left|\xi_{1}\right| \leq k,\left|\xi_{2}\right| \leq k$ et $\left|\xi_{1}-\xi_{2}\right| \geq \delta$ est compact et $A$ est continue en $(s, \xi)$ pour presque tout $x$, donc $\left[A\left(x, s, \xi_{1}\right)-A\left(x, s, \xi_{2}\right)\right]\left(\xi_{1}-\xi_{2}\right)$ atteint sur ce compact son minimum que nous noterons $\gamma(x)$, qui vérifie $\gamma(x)>0$ presque partout. De plus grâce à un résultat d'intégration, il existe $\varepsilon^{\prime}>0$ tel que

$$
\int_{A_{6}} \gamma \leq \varepsilon^{\prime} \Longrightarrow \operatorname{mes}\left(A_{6}\right) \leq \varepsilon
$$

il suffit donc de montrer que $\int_{A_{6}} \gamma \leq \varepsilon^{\prime}$. Par définition de $\gamma$, nous avons

$$
\begin{aligned}
& \int_{A_{6}} \gamma \leq \\
& \int_{A_{6}}\left(A\left(x, u_{n}, \nabla u_{n}\right)-A\left(x, u_{n}, \nabla T_{k}(u)\right)\right)\left(\nabla u_{n}-\nabla T_{k}(u)\right) 1_{\left\{\left|u_{n}-T_{k}(u)\right| \leq \eta\right\}}
\end{aligned}
$$

car sur $A_{6},\left|u_{n}-T_{k}(u)\right|=\left|u_{n}-u\right| \leq \eta$. De plus, le terme intégré est positif et

$$
\nabla T_{\eta}\left(u_{n}-T_{k}(u)\right)=\left(\nabla u_{n}-\nabla T_{k}(u)\right) 1_{\left\{\left|u_{n}-T_{k}(u)\right| \leq \eta\right\}} ;
$$

nous avons donc

$$
\begin{aligned}
\int_{A_{6}} \gamma \leq & \int_{\Omega}\left(A\left(x, u_{n}, \nabla u_{n}\right)-A\left(x, u_{n}, \nabla T_{k}(u)\right)\right) \nabla T_{\eta}\left(u_{n}-T_{k}(u)\right) \\
\leq & \int_{\Omega} A\left(x, u_{n}, \nabla u_{n}\right) \nabla T_{\eta}\left(u_{n}-T_{k}(u)\right) \\
& -\int_{\Omega} A\left(x, u_{n}, \nabla T_{k}(u)\right) \nabla T_{\eta}\left(u_{n}-T_{k}(u)\right) \\
- & -303-
\end{aligned}
$$




\section{Alain Prignet}

et, si l'on choisit $\varphi=T_{\eta}\left(u_{n}-T_{k}(u)\right) \in W^{1, p}(\Omega) \cap L^{\infty}(\Omega)$ dans (2.3), on obtient

$$
\left|\int_{\Omega} A\left(x, u_{n}, \nabla u_{n}\right) \nabla T_{\eta}\left(u_{n}-T_{k}(u)\right)\right| \leq \eta\left(\left\|f_{n}\right\|_{L^{1}}+\left\|g_{n}\right\|_{L^{1}}\right) \leq \eta C .
$$

Il reste donc à majorer l'autre intégrale : pour $\left|u_{n}\right| \geq k+\eta$, on a $\left|u_{n}-T_{k}(u)\right| \geq \eta$ d'où $\nabla T_{\eta}\left(u_{n}-T_{k}(u)\right)=0$ si bien que

$$
\begin{aligned}
& \int_{\Omega} A\left(x, u_{n}, \nabla T_{k}(u)\right) \nabla T_{\eta}\left(u_{n}-T_{k}(u)\right)= \\
& \quad=\int_{\Omega} A\left(x, T_{k+\eta}\left(u_{n}\right), \nabla T_{k}(u)\right) \nabla T_{\eta}\left(T_{k+\eta}\left(u_{n}\right)-T_{k}(u)\right) .
\end{aligned}
$$

Soit $h>0$; choisissons maintenant $\varphi=T_{h}\left(u_{n}\right) \in W^{1, p}(\Omega) \cap L^{\infty}(\Omega)$ dans (2.3), alors

$$
\left|\int_{\Omega} A\left(x, u_{n}, \nabla u_{n}\right) \nabla T_{h}\left(u_{n}\right)\right| \leq h\left(\left\|f_{n}\right\|_{L^{1}}+\left\|g_{n}\right\|_{L^{1}}\right) \leq h C
$$

et par coercitivité

$$
\begin{aligned}
\int_{\Omega} A\left(x, u_{n}, \nabla u_{n}\right) \nabla T_{h}\left(u_{n}\right) & =\int_{\Omega} A\left(x, u_{n}, \nabla u_{n}\right) \nabla u_{n} \mathbf{1}_{\left|u_{n}\right| \leq h} \\
& \geq \alpha \int_{\Omega}\left|\nabla u_{n}\right|^{p} \mathbf{1}_{\left|u_{n}\right| \leq h}=\alpha \int_{\Omega}\left|\nabla T_{h}\left(u_{n}\right)\right|^{p} ;
\end{aligned}
$$

l'inégalité de Poincaré avec moyenne, nous donne alors

$$
\begin{aligned}
\left\|T_{h}\left(u_{n}\right)\right\|_{W^{1, p}}^{p} & \leq C\left(\int_{\Omega}\left|\nabla T_{h}\left(u_{n}\right)\right|^{p}+|\widetilde{u}|^{p}\right) \\
& \leq C\left(\frac{h C}{\alpha}+|\widetilde{u}|^{p}\right) ;
\end{aligned}
$$

$T_{h}\left(u_{n}\right)$ est donc borné dans $W^{1, p}(\Omega)$ pour tout $h$. Aussi pour $h=k+\eta$, $T_{k+\eta}\left(u_{n}\right)$ tend, à une sous-suite près, vers $T_{k+\eta}(u)$ faiblement dans $W^{1, p}(\Omega)$ et donc fortement dans $L^{p}(\Omega)$ et presque partout quand $n \rightarrow+\infty$. Donc $A\left(x, T_{k+\eta}\left(u_{n}\right), \nabla T_{k}(u)\right)$ converge p.p. et $\left|A\left(x, T_{k+\eta}\left(u_{n}\right), \nabla T_{k}(u)\right)\right|^{p^{\prime}}$ est équi-intégrable, aussi grâce au théorème de Vitali, $A\left(x, T_{k+\eta}\left(u_{n}\right), \nabla T_{k}(u)\right)$ converge fortement dans $L^{p^{\prime}}(\Omega)$ vers $A\left(x, T_{k+\eta}(u), \nabla T_{k}(u)\right)$. Comme $\mathbf{1}_{\left\{\left|T_{k+\eta}\left(u_{n}\right)-T_{k}(u)\right| \leq \eta\right\}}$ tend presque partout vers $\mathbf{1}_{\left\{\left|T_{k+\eta}(u)-T_{k}(u)\right| \leq \eta\right\}}$ et est 
Conditions aux limites non homogènes pour des problèmes elliptiques

borné, le théorème de convergence dominée de Lebesgue donne la convergence forte de

$$
A\left(x, T_{k+\eta}\left(u_{n}\right), \nabla T_{k}(u)\right) \mathbf{1}_{\left\{\left|T_{k+\eta}\left(u_{n}\right)-T_{k}(u)\right| \leq \eta\right\}}
$$

vers

$$
A\left(x, T_{k+\eta}(u), \nabla T_{k}(u)\right) \mathbf{1}_{\left\{\left|T_{k+\eta}(u)-T_{k}(u)\right| \leq \eta\right\}}
$$

dans $L^{p^{\prime}}(\Omega)$.

Comme $T_{k+\eta}\left(u_{n}\right)$ converge faiblement dans $W^{1, p}(\Omega), \nabla\left(T_{k+\eta}\left(u_{n}\right)-\right.$ $\left.T_{k}(u)\right)$ converge faiblement vers $\nabla\left(T_{k+\eta}(u)-T_{k}(u)\right)$ dans $L^{p}(\Omega)$ pour $n \rightarrow+\infty$ donc

$$
\begin{gathered}
\lim _{n \rightarrow+\infty} \int_{\Omega} A\left(x, T_{k+\eta}\left(u_{n}\right), \nabla T_{k}(u)\right) \nabla T_{\eta}\left(T_{k+\eta}\left(u_{n}\right)-T_{k}(u)\right)= \\
=\int_{\Omega} A\left(x, T_{k+\eta}(u), \nabla T_{k}(u)\right) \nabla T_{\eta}\left(T_{k+\eta}(u)-T_{k}(u)\right) .
\end{gathered}
$$

Or $\nabla T_{\eta}\left(T_{k+\eta}(u)-T_{k}(u)\right) \rightarrow 0$ p.p. quand $\eta \rightarrow 0$, et pour $\eta \leq 1$

$$
\left|\nabla T_{\eta}\left(T_{k+\eta}(u)-T_{k}(u)\right)\right| \leq\left|\nabla T_{1}\left(T_{k+1}(u)-T_{k}(u)\right)\right| \in L^{p}(\Omega)
$$

et

$$
\begin{aligned}
& \left|\nabla T_{\eta}\left(T_{k+\eta}(u)-T_{k}(u)\right)\right| \leq \\
& \quad \leq \beta\left(b(x)+\left|T_{k+1}(u)\right|^{p-1}+\left|\nabla T_{k}(u)\right|^{p-1}\right) \in L^{p^{\prime}}(\Omega),
\end{aligned}
$$

donc par convergence dominée, l'intégrale du second membre tend vers 0 pour $\eta \rightarrow 0$.

Fixons $\eta<\varepsilon^{\prime} / 2 C$ tel que

$$
\left|\int_{\Omega} A\left(x, T_{k+\eta}(u), \nabla T_{k}(u)\right) \nabla T_{\eta}\left(T_{k+\eta}(u)-T_{k}(u)\right)\right| \leq \frac{\varepsilon^{\prime}}{4},
$$

soit alors $n_{2}$ tel que pour tout $n \geq n_{2}$

$$
\begin{gathered}
\mid \int_{\Omega} A\left(x, T_{k+\eta}\left(u_{n}\right), \nabla T_{k}(u)\right) \nabla T_{\eta}\left(T_{k+\eta}\left(u_{n}\right)-T_{k}(u)\right)+ \\
-\int_{\Omega} A\left(x, T_{k+\eta}(u), \nabla T_{k}(u)\right) \nabla T_{\eta}\left(T_{k+\eta}(u)-T_{k}(u)\right) \mid \leq \frac{\varepsilon^{\prime}}{4} . \\
-305-
\end{gathered}
$$


Comme $\nabla T_{\eta}\left(T_{k+\eta}\left(u_{n}\right)-T_{k}(u)\right)=\nabla T_{\eta}\left(u_{n}-T_{k}(u)\right)$, on obtient

$$
\left|\int_{\Omega} A\left(x, u_{n}, \nabla T_{k}(u)\right) \nabla T_{\eta}\left(u_{n}-T_{k}(u)\right)\right| \leq \frac{\varepsilon^{\prime}}{4}
$$

alors

$$
\int_{A_{6}} \gamma \leq C \frac{\varepsilon^{\prime}}{2 C}+\frac{\varepsilon^{\prime}}{2}=\varepsilon^{\prime}
$$

le choix de $\varepsilon^{\prime}$ entraîne donc

$$
\operatorname{mes}\left(A_{6}\right) \leq \varepsilon
$$

Ainsi $\eta$ étant fixé, les majorations de $\operatorname{mes}\left(A_{5}\right)$ et de $\operatorname{mes}\left(A_{6}\right)$ nous donnent $n_{1}$ et $n_{2}$ tels que pour $n \geq \max \left(n_{1}, n_{2}\right)$ on ait

$$
\operatorname{mes}\left(\left\{\left|\nabla u_{n}-\nabla u_{m}(x)\right| \geq \delta\right\}\right) \leq 6 \varepsilon .
$$

La convergence en mesure est donc démontrée.

Étape 3

Montrons que $u$ est solution de (2.1). Soit $\varphi \in W^{1, r}(\Omega)$ avec $r>N$, alors $\varphi \in W^{1, p}(\Omega) \cap L^{\infty}(\Omega)$ donc, par définition de $u_{n}$,

$$
\int_{\Omega} A\left(x, u_{n}, \nabla u_{n}\right) \nabla \varphi=\int_{\partial \Omega} \varphi g_{n}+\int_{\Omega} \varphi f_{n} .
$$

Comme $f_{n}$ et $g_{n}$ convergent dans $M(\bar{\Omega}) *$-faible et $M(\partial \Omega) *$-faible respectivement et que $\varphi \in W^{1, p}(\Omega) \subset C(\bar{\Omega}), \int_{\partial \Omega} \varphi g_{n}+\int_{\Omega} \varphi f_{n}$ converge vers $\int_{\partial \Omega} \varphi \mathrm{d} g+\int_{\Omega} \varphi \mathrm{d} f$. Or nous avons vu que $u_{n}$ et $\nabla u_{n}$ sont bornés dans $L^{q}(\Omega)$ pour $q<(p-1) N /(N-1)$; la condition de croissance sur $A$ entraîne donc que $A\left(x, u_{n}, \nabla u_{n}\right)$ est borné dans $L^{q}(\Omega)$ pour $q<N /(N-1)$. Soit $s<q$; l'inégalité de Hölder nous donne alors pour $E$ mesurable

$$
\begin{aligned}
& \int_{E}\left|A\left(x, u_{n}, \nabla u_{n}\right)-A(x, u, \nabla u)\right|^{s} \leq \\
& \quad \leq\left(\int_{\Omega}\left|A\left(x, u_{n}, \nabla u_{n}\right)-A(x, u, \nabla u)\right|^{q}\right)^{s / q}\left(\int_{\Omega} \mathbf{1}_{E}\right)^{1-s / q} \\
& \quad \leq C \operatorname{mes}(E) .
\end{aligned}
$$


Ainsi $\left|A\left(x, u_{n}, \nabla u_{n}\right)-A(x, u, \nabla u)\right|^{s}$ est équi-intégrable et converge presque partout vers 0 , donc converge dans $L^{1}(\Omega)$, grâce au théorème de Vitali, et donc $A\left(x, u_{n}, \nabla u_{n}\right) \rightarrow A(x, u, \nabla u)$ dans $L^{s}(\Omega)$ pour $s<N /(N-1)$; ainsi

$$
\int_{\Omega} A\left(x, u_{n}, \nabla u_{n}\right) \nabla \varphi \longrightarrow \int_{\Omega} A(x, u, \nabla u) \nabla \varphi
$$

et donc

$$
\forall \varphi \in \bigcup_{r>N} W^{1, r}(\Omega), \quad \int_{\Omega} A(x, u, \nabla u) \nabla \varphi=\int_{\partial \Omega} \varphi \mathrm{d} g+\int_{\Omega} \varphi \mathrm{d} f .
$$

Remarquons que $\nabla u_{n}$ est borné dans $W^{1, q}(\Omega)$ pour $q<(p-1) N /(N-1)$. Aussi de la même façon que ci-dessus, nous pouvons montrer que $\mid \nabla\left(u_{n}-\right.$ $u)\left.\right|^{q}$ est équi-intégrable et converge presque partout et donc, grâce au théorème de Vitali, que $\nabla u_{n} \rightarrow \nabla u$ dans $L^{q}(\Omega)$ pour $q<(p-1) N /(N-1)$.

\section{Conditions aux limites de Fourier}

Le problème de Fourier consiste à chercher $u$ telle que

$$
\begin{array}{cc}
-\operatorname{div}(A(x, u, \nabla u))=f & \operatorname{dans} \Omega \\
A(x, u, \nabla u) \cdot n+\lambda u=g & \text { sur } \partial \Omega
\end{array}
$$

avec $f \in M(\bar{\Omega}), g \in M(\partial \Omega)$ et $\lambda>0$. On résoudra (3.1) au sens faible suivant : on cherche

$$
u \in \bigcap_{q<(p-1) N /(N-1)} W^{1, q}(\Omega)
$$

tel que

$\forall \varphi \in \bigcup_{r>N} W^{1, r}(\Omega), \quad \int_{\Omega} A(x, u, \nabla u) \nabla \varphi+\lambda \int_{\partial \Omega} u \varphi=\int_{\partial \Omega} \varphi \mathrm{d} g+\int_{\Omega} \varphi \mathrm{d} f$.

THÉORÈME .- Soient $f \in M(\bar{\Omega})$ (ne chargeant pas le bord), $g \in$ $M(\partial \Omega), \Omega$ un ouvert borné régulier, et soit $A$ vérifiant les hypothèses (H), alors le problème (3.1) admet une solution. 


\section{Alain Prignet}

Avant de démontrer ce résultat rappelons une inégalité de type Poincaré et une inégalité de type Poincaré-Sobolev (que nous démontrerons par souci de clarté).

LeMme . - Il existe $C_{1}>0$ tel que pour tout $u \in W^{1,1}(\Omega)$ on ait

$$
\int_{\Omega}|u| \leq C_{1}\left(\int_{\Omega}|\nabla u|+\int_{\partial \Omega}|u|\right)
$$

et il existe $C_{2}$ tel que pour tout $u \in W^{1, q}(\Omega), 1<q<N$, on ait

$$
\left(\int_{\Omega}|u|^{q^{*}}\right)^{q / q^{*}} \leq C_{2}\left(\left(\int_{\partial \Omega}|u|\right)^{q}+\int_{\Omega}|\nabla u|^{q}\right) .
$$

Démonstration du lemme. - Montrons la première inégalité par l'absurde. Supposons qu'il existe une suite $\left(u_{n}\right)$ telle que $\int_{\Omega}\left|u_{n}\right|=|\Omega|$ et $\int_{\Omega}\left|\nabla u_{n}\right|+\int_{\partial \Omega}\left|u_{n}\right| \rightarrow 0$; appliquons alors l'inégalité de Poincaré avec moyenne

$$
\int_{\Omega}\left|u_{n}-1\right| \leq C \int_{\Omega}\left|\nabla u_{n}\right| \longrightarrow 0
$$

donc $u_{n} \rightarrow 1$ dans $L^{1}(\Omega)$ et dans $W^{1,1}(\Omega)$; or $\int_{\partial \Omega} 1 \neq 0$ et l'application trace est continue, il y a donc une contradiction.

Montrons maintenant la seconde inégalité. Soit $q \geq 1$; alors, d'après les inégalités de Poincaré avec moyenne et de Sobolev, il existe $C>0$ tel que pour $u \in W^{1, q}(\Omega)$ avec $\int_{\Omega} u=\widetilde{u}|\Omega|$, on ait

$$
\|u-\tilde{u}\|_{L^{q^{*}}} \leq C\|\nabla u\|_{L^{q}}
$$

soit

$$
\int_{\Omega}|u|^{q^{*}} \leq C \int_{\Omega}|\widetilde{u}|^{q^{*}}+\left(\int_{\Omega}|\nabla u|^{q}\right)^{q^{*} / q}
$$

or

$$
\int_{\Omega}|\widetilde{u}|^{q^{*}}=|\widetilde{u}|^{q^{*}}|\Omega| \text { et }|\widetilde{u}|^{q^{*}}|\Omega|^{q^{*}} \leq\left(\int_{\Omega}|u|\right)^{q^{*}}
$$

donc

$$
\begin{aligned}
\int_{\Omega}|\widetilde{u}|^{q^{*}} & =C\left(\int_{\Omega}|u|\right)^{q^{*}} \\
& -308-
\end{aligned}
$$


d'où

$$
\int_{\Omega}|u|^{q^{*}} \leq C\left(\int_{\Omega}|u|\right)^{q^{*}}+\left(\int_{\Omega}|\nabla u|^{q}\right)^{q^{*} / q} .
$$

Appliquons alors le lemme puisque $u \in W^{1,1}$

$$
\int_{\Omega}|u| \leq C\left(\int_{\Omega}|\nabla u|+\int_{\partial \Omega}|u|\right)
$$

et

$$
\int_{\Omega}|\nabla u| \leq C\left(\int_{\Omega}|\nabla u|^{q}\right)^{1 / q} \quad \text { (inégalité de Hölder) }
$$

donc

$$
\int_{\Omega}|u|^{q^{*}} \leq C\left(\left(\int_{\partial \Omega}|u|\right)^{q^{*}}+\left(\int_{\Omega}|\nabla u|^{q}\right)^{q}\right)^{q^{*} / q}
$$

ce résultat nous donne

$$
\left(\int_{\Omega}|u|^{q^{*}}\right)^{q / q^{*}} \leq C\left(\left(\int_{\partial \Omega}|u|\right)^{q}+\int_{\Omega}|\nabla u|^{q}\right)
$$

qui est bien l'inégalité demandée.

Démonstration du théorème. - Soient $\left(f_{n}\right) \in W^{-1, p^{\prime}}(\Omega) \cap L^{1}(\Omega)$ et $\left(g_{n}\right) \in\left(W^{1-1 / p, p}(\partial \Omega)\right)^{\prime} \cap L^{1}(\partial \Omega)$ tels que

$$
\left\|f_{n}\right\|_{L^{1}} \leq\|f\|_{M(\bar{\Omega})} \quad \text { et } \quad\left\|g_{n}\right\|_{L^{1}} \leq\|g\|_{M(\partial \Omega)},
$$

$f_{n} \rightarrow f$ dans $M(\bar{\Omega}) *$-faible et $g_{n} \rightarrow g$ dans $M(\partial \Omega) *$-faible. Soit $u_{n} \in$ $W^{1, p}(\Omega)$ une solution de (3.1) avec $f=f_{n}$ et $g=g_{n}$ (Leray-Lions [7]). Elle vérifie

$$
\begin{aligned}
& \forall \varphi \in W^{1, p} \cap L^{\infty}(\Omega), \\
& \qquad \int_{\Omega} A\left(x, u_{n}, \nabla u_{n}\right) \nabla \varphi+\lambda \int_{\partial \Omega} u_{n} \varphi=\int_{\partial \Omega} \varphi g_{n}+\int_{\Omega} \varphi f_{n} .
\end{aligned}
$$

Étape 1

Montrons que $u_{n}$ est borné dans $W^{1, q}(\Omega)$ pour $q<(p-1) N /(N-1)$. La méthode est la même que pour les conditions de Neumann, mais l'inégalité de Poincaré-Sobolev que nous venons de démontrer fait intervenir $\int_{\partial \Omega}\left|u_{n}\right|$. 
Il nous faut donc une estimation de cette intégrale. Comme $T_{k}\left(u_{n}\right) \in$ $W^{1, p}(\Omega) \cap L^{\infty}(\Omega)$, nous pouvons choisir $\varphi=T_{k}\left(u_{n}\right)$ dans (3.2), ce qui donne

$$
\begin{gathered}
\int_{\Omega} A\left(x, u_{n}, \nabla u_{n}\right) \nabla u_{n} T_{k}^{\prime}\left(u_{n}\right)+\lambda \int_{\partial \Omega} u_{n} T_{k}\left(u_{n}\right)= \\
=\int_{\partial \Omega} T_{k}\left(u_{n}\right) g_{n}+\int_{\Omega} T_{k}\left(u_{n}\right) f_{n} .
\end{gathered}
$$

Or $A\left(x, u_{n}, \nabla u_{n}\right) \nabla u_{n} \geq 0, T_{k}^{\prime}(x) \geq 0$ et $\left|T_{k}\right| \leq k$. Donc

$$
\lambda \int_{\partial \Omega} u_{n} T_{k}\left(u_{n}\right) \leq k\left(\left\|g_{n}\right\|_{L^{1}}+\left\|f_{n}\right\|_{L^{1}}\right)
$$

soit

$$
\lambda \int_{\partial \Omega} u_{n} \frac{1}{k} T_{k}\left(u_{n}\right) \leq C
$$

Or $\lim _{k \rightarrow 0} x T_{k}(x) / k=|x|$ pour $x \neq 0$. Donc en passant à la limite pour $k \rightarrow 0$ (par convergence dominée), on a

$$
\int_{\partial \Omega}\left|u_{n}\right| \leq \frac{C}{\lambda}
$$

Comme pour les conditions de Neumann, choisissons $\varphi=\psi_{m}\left(u_{n}\right)$ dans (3.2). Or $\lambda>0$ et $x \psi_{m}(x) \geq 0$ donc $\lambda \int_{\partial \Omega} u_{n} \psi_{m}\left(u_{n}\right) \geq 0$. Nous obtenons alors

$$
\alpha \int_{\Omega}\left|\nabla u_{n}\right|^{p} \psi_{m}^{\prime}\left(u_{n}\right) \leq\left\|f_{n}\right\|_{L^{1}}+\left\|g_{n}\right\|_{L^{1}} .
$$

Comme dans le cas de Neumann, on peut montrer que

$$
\int_{\Omega}\left|\nabla u_{n}\right|^{q} \leq C \varepsilon^{(p-q) / p}\left(\int_{\Omega}\left|u_{n}\right|^{q^{*}}\right)^{(p-q) / p}+C ;
$$

donc, grâce au lemme, à (3.3) et pour $\varepsilon$ petit, on a

$$
\left(\int_{\Omega}\left|u_{n}\right|^{q^{*}}\right)^{1 / q^{*}} \leq \frac{1}{2}\left(\int_{\Omega}\left|u_{n}\right|^{q^{*}}\right)^{(p-q) / p q}+C+\frac{C}{\lambda} ;
$$

on conclut de la même façon que pour les conditions de Neumann. 


\section{Étape 2}

Il faut ici aussi montrer la convergence presque partout de $\nabla u_{n}$. On procède comme précédemment : on construit de même $A_{1}$ à $A_{6}$ et seule la majoration de $\operatorname{mes}\left(A_{6}\right)$ fait intervenir (3.2), les autres majorations s'appliquent de façon identique. Pour celle de $A_{6}$, on peut remarquer que si l'on choisit $\varphi=T_{\eta}\left(u_{n}-T_{k}(u)\right)$ dans (3.2) on obtient

$$
\begin{gathered}
\int_{\Omega} A\left(x, u_{n}, \nabla u_{n}\right) \nabla T_{\eta}\left(u_{n}-T_{k}(u)\right)+\lambda \int_{\Omega} u_{n} T_{\eta}\left(u_{n}-T_{k}(u)\right)= \\
=\int_{\Omega} f_{n} T_{\eta}\left(u_{n}-T_{k}(u)\right)+\int_{\partial \Omega} g_{n} T_{\eta}\left(u_{n}-T_{k}(u)\right) .
\end{gathered}
$$

Notons le second membre $C_{\eta, n}$, nous obtenons alors

$$
\begin{gathered}
\int_{\Omega}\left(A\left(x, u_{n}, \nabla u_{n}\right)-A\left(x, u_{n}, \nabla T_{k}(u)\right)\right) \nabla T_{\eta}\left(u_{n}-T_{k}(u)\right)+ \\
\quad+\lambda \int_{\Omega}\left(u_{n}-T_{k}(u)\right) T_{\eta}\left(u_{n}-T_{k}(u)\right) \\
=C_{\eta, n}+\lambda \int_{\Omega} T_{k}(u) T_{\eta}\left(u_{n}-T_{k}(u)\right)+ \\
\quad-\int_{\Omega} A\left(x, u_{n}, \nabla T_{k}(u)\right) \nabla T_{\eta}\left(u_{n}-T_{k}(u)\right) .
\end{gathered}
$$

De plus, nous avons $\left(u_{n}-T_{k}(u)\right) T_{\eta}\left(u_{n}-T_{k}(u)\right) \geq 0,\left|C_{\eta, n}\right| \leq \eta C$ et $\left|\int_{\Omega} T_{k}(u) T_{\eta}\left(u_{n}-T_{k}(u)\right)\right| \leq k \eta C$, donc

$$
\begin{array}{r}
\int_{\Omega}\left(A\left(x, u_{n}, \nabla u_{n}\right)-A\left(x, u_{n}, \nabla T_{k}(u)\right)\right) \nabla T_{\eta}\left(u_{n}-T_{k}(u)\right) \leq \\
\leq \eta C(1+\lambda k)+\left|\int_{\Omega} A\left(x, u_{n}, \nabla T_{k}(u)\right) \nabla T_{\eta}\left(u_{n}-T_{k}(u)\right)\right|
\end{array}
$$

où $k$ a été fixé pour que les mesures de $A_{1}$ à $A_{4}$ soient inférieures à $\varepsilon$.

Si l'on choisit $\varphi=T_{h}\left(u_{n}\right)$ dans (3.2), comme $u_{n} T_{h}\left(u_{n}\right) \geq 0$, on obtient

$$
\int_{\Omega} A\left(x, u_{n}, \nabla u_{n}\right) \nabla T_{h}\left(u_{n}\right) \leq h C .
$$

Or nous avons vu que $\int_{\partial \Omega}\left|u_{n}\right|$ est borné, donc $\int_{\partial \Omega} T_{k}\left(u_{n}\right)$ l'est aussi. On peut donc, grâce au lemme, démontrer comme précédemment que $T_{h}\left(u_{n}\right)$ est borné dans $W^{1, p}(\Omega)$ et on peut choisir $\eta<\varepsilon^{\prime} / 2 C(1+\lambda k)$ tel que

$$
\left|\int_{\Omega} A\left(x, u_{n}, \nabla T_{k}(u)\right) \nabla T_{\eta}\left(u_{n}-T_{k}(u)\right)\right| \leq \frac{\varepsilon^{\prime}}{2}
$$


pour $n$ assez grand, alors

$$
\int_{A_{6}} \gamma \leq \int_{\Omega}\left(A\left(x, u_{n}, \nabla u_{n}\right)-A\left(x, u_{n}, \nabla T_{k}(u)\right)\right) \nabla T_{\eta}\left(u_{n}-T_{k}(u)\right) \leq \varepsilon^{\prime}
$$

ce qui permet d'achever cette étape de la même façon.

Étape 3

Comme pour les conditions aux limites de Neumann on a, pour $\varphi \in$ $W^{1, r}(\Omega)$ avec $r>N$, les convergences de $\int_{\Omega} A\left(x, u_{n}, \nabla u_{n}\right) \nabla \varphi, \int_{\Omega} \varphi f_{n}$ et $\int_{\partial \Omega} \varphi g_{n}$; il reste donc à montrer celle de $\int_{\partial \Omega} u_{n} \varphi$. Comme $u_{n} \rightarrow u$ dans $W^{1, q}(\Omega)$ pour $q<(p-1) N /(N-1)$, la trace de $u_{n}$ sur $\partial \Omega$ converge vers celle de $u$ dans $W^{1-1 / q, q}(\partial \Omega)$ et donc dans $L^{q}(\partial \Omega)$; comme $\varphi \in W^{1, r}(\Omega)$ pour $r>N, \varphi \in C(\bar{\Omega})$ donc $\varphi \in L^{\infty}(\partial \Omega)$ et on obtient

$$
\int_{\partial \Omega} u_{n} \varphi \longrightarrow \int_{\partial \Omega} u \varphi
$$

ce qui permet de conclure que $u$ est solution de (3.1).

\section{Conditions aux limites de Dirichlet}

Le problème de Dirichlet consiste à chercher $u$ tel que

$$
\begin{aligned}
-\operatorname{div}(A(x, u, \nabla u)) & =f & & \operatorname{dans} \Omega \\
u & =g & & \operatorname{sur} \partial \Omega
\end{aligned}
$$

avec $f \in M(\bar{\Omega})$ et $g \in W^{1-1 / p, p}(\partial \Omega)$. On résoudra (4.1) au sens faible suivant : on cherche

$$
u \in \bigcap_{g<(p-1) N /(N-1)} W^{1, q}(\Omega)
$$

tel que, pour $\widetilde{g} \in W^{1, p}(\Omega)$, un relèvement de $g$, on ait

$$
u-\tilde{g} \in \bigcap_{q<(p-1) N /(N-1)} W_{0}^{1, q}(\Omega)
$$

et

$$
\forall \varphi \in \bigcup_{r>N} W_{0}^{1, r}(\Omega), \quad \int_{\Omega} A(x, u, \nabla u) \nabla \varphi=\int_{\Omega} \varphi \mathrm{d} f
$$


THÉORÈME .- Soient $f \in M(\bar{\Omega}), g \in W^{1-1 / p, p}(\partial \Omega), \Omega$ un ouvert borné régulier, et soit $A$ vérifiant les hypothèses (H), alors le problème (4.1) admet une solution.

Démonstration. - Soient $\left(f_{n}\right) \in W^{-1, p^{\prime}}(\Omega) \cap L^{1}(\Omega)$ tel que $\left\|f_{n}\right\|_{L^{1}} \leq$ $\|f\|_{M(\bar{\Omega})}$ et $f_{n} \rightarrow f$ dans $M(\bar{\Omega}) *$-faible. Soit $u_{n} \in W^{1, p}(\Omega)$ une solution de (4.1) avec $f=f_{n}$ (Leray-Lions [7]), elle vérifie

$$
\forall \varphi \in W_{0}^{1, r} \cap L^{\infty}(\Omega), \quad \int_{\Omega} A\left(x, u_{n}, \nabla u_{n}\right) \nabla \varphi=\int_{\Omega} \varphi f_{n},
$$

et $u_{n}-\tilde{g} \in W_{0}^{1, p}(\Omega)$.

\section{Étape 1}

Nous montrons, là encore, que $u_{n}$ est borné dans $W^{1, q}(\Omega)$ pour $q<$ $(p-1) N /(N-1)$. La démonstration ne peut pas être faite ici avec $\varphi=$ $\psi_{m}\left(u_{n}-\widetilde{g}\right)$; aussi, nous allons adopter une variante de la démonstration correspondant aux conditions aux limites de Neumann.

Soit $k \in \mathbb{N}$; choisissons $\varphi=T_{k}\left(u_{n}-\widetilde{g}\right) \in W_{0}^{1, p} \cap L^{\infty}(\Omega)$, alors

$$
\int_{\Omega} A\left(x, u_{n}, \nabla u_{n}\right) \nabla\left(u_{n}-\widetilde{g}\right) \mathbf{1}_{\left\{\left|u_{n}-\tilde{g}\right| \leq k\right\}}=\int_{\Omega} T_{k}\left(u_{n}-\widetilde{g}\right) f_{n}
$$

donc

$$
\begin{aligned}
& \int_{\Omega} A\left(x, u_{n}, \nabla u_{n}\right) \nabla u_{n} \mathbf{1}_{\left\{\left|u_{n}-\tilde{g}\right| \leq k\right\}}= \\
& \quad=\int_{\Omega} T_{k}\left(u_{n}-\widetilde{g}\right) f_{n}+\int_{\Omega} A\left(x, u_{n}, \nabla u_{n}\right) \nabla \widetilde{g} \mathbf{1}_{\left\{\left|u_{n}-\tilde{g}\right| \leq k\right\}} ;
\end{aligned}
$$

nous avons, par coercivité et avec la condition de croissance de $A$,

$$
\begin{aligned}
& \alpha \int_{\Omega}\left|\nabla u_{n}\right|^{p} \mathbf{1}_{\left\{\left|u_{n}-\tilde{g}\right| \leq k\right\}} \leq \\
& \quad \leq k\left\|f_{n}\right\|_{L^{1}}+\beta \int_{\Omega}\left(b(x)+\left|u_{n}\right|^{p-1}+\left|\nabla\left(u_{n}\right)\right|^{p-1}\right)|\nabla \widetilde{g}| \mathbf{1}_{\left\{\left|u_{n}-\tilde{g}\right| \leq k\right\}}
\end{aligned}
$$

et, grâce à l'inégalité de Hölder,

$$
\begin{gathered}
\int_{\Omega}\left(b(x)+\left|u_{n}\right|^{p-1}+\left|\nabla u_{n}\right|^{p-1}\right)|\nabla \widetilde{g}| \mathbf{1}_{\left\{\left|u_{n}-\tilde{g}\right| \leq k\right\}} \leq \\
\leq\|\tilde{g}\|_{W^{1, p}}\left(\int_{\Omega}\left(b(x)^{p^{\prime}}+\left|u_{n}\right|^{p}+\left|\nabla u_{n}\right|^{p}\right)\left(\mathbf{1}_{\left\{\left|u_{n}-\tilde{g}\right| \leq k\right\}}\right)\right)^{(p-1) / p} \\
-313-
\end{gathered}
$$




\section{Alain Prignet}

car

$$
\left(\mathbf{1}_{\left\{\left|u_{n}-\tilde{g}\right| \leq k\right\}}\right) p /(p-1)=\mathbf{1}_{\left\{\left|u_{n}-\tilde{g}\right| \leq k\right\}}
$$

L'inégalité de Poincaré nous donne, appliquée à $T_{k}\left(u_{n}-\widetilde{g}\right) \in W_{0}^{1, p}(\Omega)$,

$$
\int_{\Omega}\left|u_{n}-\tilde{g}\right|^{p} \mathbf{1}_{\left\{\left|u_{n}-\tilde{g}\right| \leq k\right\}} \leq \int_{\Omega}\left|T_{k}\left(u_{n}-\tilde{g}\right)\right|^{p} \leq C \int_{\Omega}\left|\nabla\left(u_{n}-\tilde{g}\right)\right|^{p} \mathbf{1}_{\left\{\left|u_{n}-\tilde{g}\right| \leq k\right\}}
$$

si bien que

$$
\int_{\Omega}\left|u_{n}\right|^{p} \mathbf{1}_{\left\{\left|u_{n}-\tilde{g}\right| \leq k\right\}} \leq C \int_{\Omega}\left(|\tilde{g}|^{p}+|\nabla \tilde{g}|^{p}\right)+C \int_{\Omega}\left|\nabla u_{n}\right|^{p} \mathbf{1}_{\left\{\left|u_{n}-\tilde{g}\right| \leq k\right\}} ;
$$

alors on a

$$
\begin{aligned}
& \alpha \int_{\Omega}\left|\nabla u_{n}\right|^{p} \mathbf{1}_{\left\{\left|u_{n}-\tilde{g}\right| \leq k\right\}} \leq \\
& \leq k\|f\|_{M(\bar{\Omega})}+\beta C\|\tilde{g}\|_{W^{1, p^{\prime}}}\left(\|b\|_{L^{p^{\prime}}}+\left(\|\tilde{g}\|_{W^{1, p}}\right)^{p-1}\right)+ \\
& \quad+2 \beta C\|\tilde{g}\|_{W^{1, p}}\left(\int_{\Omega}\left|\nabla u_{n}\right|^{p} \mathbf{1}_{\left\{\left|u_{n}-\tilde{g}\right| \leq k\right\}}\right)^{(p-1) / p}
\end{aligned}
$$

donc

$$
\frac{1}{k} \int_{\Omega}\left|\nabla u_{n}\right|^{p} \mathbf{1}_{\left\{\left|u_{n}-\tilde{g}\right| \leq k\right\}} \leq C+C\left(\frac{1}{k} \int_{\Omega}\left|\nabla u_{n}\right|^{p} \mathbf{1}_{\left\{\left|u_{n}-\tilde{g}\right| \leq k\right\}}\right)^{(p-1) / p}
$$

où $(p-1) / p<1$. Donc, il existe $C$ indépendant de $n$ et de $k$ tel que

$$
\int_{\Omega}\left|\nabla u_{n}\right|^{p} \mathbf{1}_{\left\{\left|u_{n}-\tilde{g}\right| \leq k\right\}} \leq C k .
$$

Or

$$
\int_{\Omega}|\nabla \widetilde{g}|^{p} \mathbf{1}_{\left\{\left|u_{n}-\tilde{g}\right| \leq k\right\}} \leq\left(\|\nabla \widetilde{g}\|_{L^{p}}\right)^{p}
$$

donc

$$
\int_{\Omega}\left|\nabla\left(u_{n}-\tilde{g}\right)\right|^{p} \mathbf{1}_{\left\{\left|u_{n}-\tilde{g}\right| \leq k\right\}} \leq C k
$$

et donc

$$
\sum_{\ell=0}^{k-1} \int_{\Omega}\left|\nabla\left(u_{n}-\tilde{g}\right)\right|^{p} 1_{\left\{\ell \leq\left|u_{n}-\tilde{g}\right| \leq \ell+k\right\}} \leq C k .
$$


Conditions aux limites non homogènes pour des problèmes elliptiques

Comme précédemment, il s'agit de majorer

$$
\begin{aligned}
& \int_{\Omega} \frac{\left|\nabla\left(u_{n}-\widetilde{g}\right)\right|^{p}}{\left(1+\left|u_{n}-\tilde{g}\right|\right)^{m+1}}= \\
& \quad=\sum_{k \in \mathbb{N}} \int_{\Omega} \frac{\left|\nabla\left(u_{n}-\widetilde{g}\right)\right|^{p}}{\left(1+\left|u_{n}-\tilde{g}\right|\right)^{m+1}} \mathbf{1}_{\left\{k \leq\left|u_{n}-\tilde{g}\right| \leq k+1\right\}} \\
& \quad \leq \sum_{k \in \mathbb{N}} \frac{1}{(1+k)^{m+1}} \int_{\Omega}\left|\nabla\left(u_{n}-\widetilde{g}\right)\right|^{p} 1_{\left\{k \leq\left|u_{n}-\tilde{g}\right| \leq k+1\right\}} .
\end{aligned}
$$

Posons

$$
a^{k}=\frac{1}{(1+k)^{m+1}} \quad \text { et } \quad b_{k}=\int_{\Omega}\left|\nabla\left(u_{n}-\widetilde{g}\right)\right|^{p} \mathbf{1}_{\left\{k \leq\left|u_{n}-\tilde{g}\right| \leq k+1\right\}},
$$

ce qui nous amène à considérer $\sum_{k \in \mathbb{N}} a_{k} b_{k}$. Notons $B_{k}=\sum_{\ell=0}^{k-1} b_{\ell}$ pour $k \geq 1$ et $B_{0}=0 ;$ alors pour $N \in \mathbb{N}$

$$
\begin{aligned}
\sum_{k=0}^{N} a_{k} b_{k} & =\sum_{k=0}^{N} a_{k}\left(B_{k+1}-B_{k}\right)=\sum_{k=0}^{N} a_{k} B_{k+1}-\sum_{k=0}^{N} a_{k} B_{k} \\
& =\sum_{k=0}^{N} a_{k} B_{k+1}-\sum_{k=0}^{N} a_{k+1} B_{k+1}+a_{N+1} B_{N+1} \\
& =\sum_{k=0}^{N}\left(a_{k}-a_{k+1}\right) B_{k+1}+a_{N+1} B_{N+1} .
\end{aligned}
$$

Or (4.4) nous donne $B_{k} \leq C k$ donc $\lim _{N \rightarrow+\infty} a_{N+1} B_{N+1}=0$ et

$$
0 \leq\left(a_{k}-a_{k+1}\right) B_{k+1} \leq \frac{C k}{(1+k)^{m+2}}+\circ\left(\frac{k}{(1+k)^{m+2}}\right) .
$$

Comme $m>0$, cette série est convergente donc la série $\sum_{k \in \mathbb{N}} a_{k} b_{k}$ est aussi convergente et il existe donc $C$ tel que

$$
\int_{\Omega} \frac{\left|\nabla\left(u_{n}-\tilde{g}\right)\right|^{p}}{\left(1+\left|u_{n}-\tilde{g}\right|\right)^{m+1}} \leq \sum_{k \in \mathbb{N}} a_{k} b_{k} \leq C .
$$

Aussi, comme pour les conditions de Neumann, on peut montrer que

$$
\left.\int_{\Omega}\left|\nabla\left(u_{n}-\widetilde{g}\right)\right|^{q} \leq\left. C \varepsilon^{(p-q) / p}\left(\int_{\Omega} \mid u_{n}-\widetilde{g}\right)\right|^{q^{*}}\right)^{(p-q) / p}+C .
$$


De plus, on a $u_{n}-\widetilde{g} \in W_{0}^{1, p}(\Omega)$ donc l'inégalité de Poincaré-Sobolev nous donne

$$
\left.\left.\left(\int_{\Omega} \mid u_{n}-\widetilde{g}\right)\right|^{q^{*}}\right)^{q / q^{*}} \leq C \int_{\Omega}\left|\nabla\left(u_{n}-\widetilde{g}\right)\right|^{q} .
$$

Pour $\varepsilon$ petit, nous obtenons

$$
\left.\left.\left.\left(\int_{\Omega} \mid u_{n}-\widetilde{g}\right)\right|^{q^{*}}\right)^{1 / q^{*}} \leq\left.\frac{1}{2}\left(\int_{\Omega} \mid u_{n}-\widetilde{g}\right)\right|^{q^{*}}\right)^{(p-q) / p q}+C
$$

et, pour $q<(p-1) N /(N-1)$, on conclut de la même façon que précédemment que $u_{n}-\tilde{g}$ est borné dans $W_{0}^{1, q}(\Omega)$; donc $u_{n}$ est borné dans $W^{1, q}(\Omega)$.

Remarque. - Au cours de cette démonstration, nous avons montré le lemme suivant qui est plus fort que celui qui est utilisé dans [2].

LEMME . - Soient $\left(v_{n}\right)$ une suite de $W_{0}^{1, p}(\Omega)$ et $C>0$ tels que

$$
\int_{\Omega}\left|\nabla v_{n}\right|^{p} \mathbf{1}_{\left\{\mid v_{n} \leq k\right\}} \leq C k
$$

pour tout $k \in \mathbb{N}$, alors $\int_{\Omega}\left|\nabla v_{n}\right|^{q} \leq C^{\prime}$ donc $\left(v_{n}\right)$ est bornée dans $W_{0}^{1, q}(\Omega)$ pour tout $q<(p-1) N /(N-1)$.

Étape 2

Il s'agit de montrer la convergence de $\nabla u_{n}$ presque partout; il suffit, ici encore, de majorer mes $\left(A_{6}\right)$.

Il faut montrer que, pour $h>0, T_{h}\left(u_{n}-\widetilde{g}\right)$ est borné dans $W_{0}^{1, p}(\Omega)$. Ceci vient presque d'être fait; en effet, pour $k=h,(4.3)$ entraîne $\int_{\Omega} \mid \nabla T_{h}\left(u_{n}-\right.$ $\widetilde{g})\left.\right|^{p} \leq C h$ et, d'autre part, $\left|T_{h}\left(u_{n}-\widetilde{g}\right)\right| \leq h$; donc, pour $h$ fixé, $T_{h}\left(u_{n}-\widetilde{g}\right)$ est borné dans $W_{0}^{1, q}(\Omega)$.

Pour majorer mes $\left(A_{6}\right)$, choisissons

$$
\varphi=T_{\eta}\left(u_{n}-\widetilde{g}-T_{k}(u-\widetilde{g})\right) \in W_{0}^{1, p}(\Omega)
$$

dans (4.2), alors

$$
\left|\int_{\Omega} A\left(x, u_{n}, \nabla u_{n}\right) \nabla T_{\eta}\left(u_{n}-\tilde{g}-T_{k}(u-\widetilde{g})\right)\right| \leq \eta C .
$$


De plus, $u_{n}=u_{n}-\widetilde{g}+\widetilde{g}$ et $\nabla T_{\eta}\left(u_{n}-\widetilde{g}-T_{k}(u-\widetilde{g})\right)=0$ si $\left|u_{n}-\widetilde{g}\right| \geq k+\eta$ donc

$$
\begin{aligned}
& \int_{\Omega} A\left(x, u_{n}, \nabla T_{k}(u)\right) \nabla T_{\eta}\left(u_{n}-\tilde{g}-T_{k}(u-\widetilde{g})\right)= \\
& =\int_{\Omega} A\left(x, T_{k+\eta}\left(u_{n}-\widetilde{g}\right)+\widetilde{g}, \nabla T_{k}(u)\right) \nabla T_{\eta}\left(T_{k+\eta}\left(u_{n}-\widetilde{g}\right)-T_{k}(u-\widetilde{g})\right) .
\end{aligned}
$$

Or nous avons montré que $T_{h}\left(u_{n}-\widetilde{g}\right)$ est borné dans $W_{0}^{1, p}(\Omega)$ donc

$$
\nabla\left(T_{k+\eta}\left(u_{n}-\widetilde{g}\right)-T_{k}(u-\widetilde{g})\right)
$$

converge faiblement dans $L^{p}(\Omega)$ vers

$$
\nabla\left(T_{k+\eta}(u-\widetilde{g})-T_{k}(u-\widetilde{g})\right) ;
$$

nous pouvons faire le même raisonnement, que celui qui a été fait pour les conditions de Neumann, avec $u_{n}-\tilde{g}$ :

$$
\mathbf{1}_{\left|T_{k+\eta}\left(u_{n}-\tilde{g}\right)-T_{k}(u-\widetilde{g})\right| \leq \eta} \text { tend p.p. vers } \mathbf{1}_{\left|T_{k+\eta}(u-\widetilde{g})-T_{k}(u-\widetilde{g})\right| \leq \eta}
$$

et

$$
A\left(x, T_{k+\eta}\left(u_{n}-\widetilde{g}\right)+\tilde{g}, \nabla T_{k}(u)\right) \quad \text { converge fortement dans } L^{p^{\prime}}(\Omega)
$$

vers

$$
A\left(x, T_{k+\eta}(u-\widetilde{g})+\tilde{g}, \nabla T_{k}(u)\right) \text { pour } n \rightarrow+\infty,
$$

enfin,

$$
\nabla T_{\eta}\left(T_{k+\eta}(u-\widetilde{g})-T_{k}(u-\widetilde{g})\right) \rightarrow 0 \quad \text { p.p. pour } \eta \rightarrow 0,
$$

on peut donc choisir $\eta<\varepsilon^{\prime} / 2 C$ tel que pour $n$ assez grand

$$
\left|\int_{\Omega} A\left(x, u_{n}, \nabla u_{n}\right) \nabla T_{\eta}\left(u_{n}-\tilde{g}-T_{k}(u-\tilde{g})\right)\right| \leq \frac{\varepsilon^{\prime}}{2}
$$

et

$$
\left|\int_{\Omega} A\left(x, u_{n}, \nabla T_{k}(u)\right) \nabla T_{\eta}\left(u_{n}-\tilde{g}-T_{k}(u-\widetilde{g})\right)\right| \leq \frac{\varepsilon^{\prime}}{2} .
$$

Donc, de même que pour le problème de Neumann, on a mes $\left(A_{6}\right) \leq \varepsilon$ pour $n$ assez grand, ce qui achève la preuve de la convergence. 
Étape 3

Comme précédemment, on peut montrer que $A\left(x, u_{n}, \nabla u_{n}\right)$ converge vers $A(x, u, \nabla u)$ dans $L^{q}(\Omega)$ pour $q<N /(N-1)$ et $\nabla \varphi \in L^{r}(\Omega)$ pour $r>N$, ce qui permet de montrer que

$$
\int_{\Omega} A\left(x, u_{n}, \nabla u_{n}\right) \nabla \varphi \longrightarrow \int_{\Omega} A(x, u, \nabla u \nabla \varphi
$$

et donc que $u$ est solution de (4.1).

\section{Remerciements}

L'auteur remercie Thierry Gallouët pour l'intérêt qu'il a porté à ce travail et pour ses remarques judicieuses.

\section{Bibliographie}

[1] Bénilan (P.), Boccardo (L.), Gallouẽt (T.), Gariepy (R.), Pierre (M.) et VASQUEZ (J.-L.) - - An $L^{1}$-theory of existence and uniqueness of solutions of nonlinear elliptic equations, Ann. Scuola Norm. Pisa. Cl. Sci. 22 (1995), pp. 241273.

[2] BocCARDO (L.) et GALLOUËT (T.) . - Nonlinear elliptic and parabolic equations involving measure data, J. Funct. Anal. 87 (1989), pp. 149-169.

[3] BOCCARDO (L.) et GALlOUËT (T.) .- Nonlinear equations with right hand side measures, Comm. P. D. E. 17 (1992), pp. 641-655.

[4] Boccardo (L.) GallouËt (T.) et Vasquez (J.-L.) .- Nonlinear equations in $\mathbb{R}^{N}$ without growth restrictions on the data, J. Diff. Eqs. 105 (1993), pp. 334-363.

[5] Det Vecchio (T.) .- Nonlinear elliptic equations with measure data, Potential Analysis 4 (1995), pp. 185-204.

[6] FabRie (P.) et GallouËt (T.) .- Modelling wells in porous media flows, en préparation.

[7] LeRAY (J.) et Lions (J.-L.) .- Quelques résultats de Višik sur les problèmes elliptiques non linéaires par les méthodes de Minty-Browder, Bull. Soc. Math. France 93 (1965), pp. 97-107.

[8] Lions (P.-L.) et Murat (F.) .- - Sur les solutions renormalisées d'équations elliptiques non linéaires, en préparation.

[9] Murat (F.) - - Soluciones renormalizadas de EDP elipticas non lineales, Prépublication de l'Université de Paris VI, France, 1993.

[10] PRIGNet (A.) .- Remarks on existence and uniqueness of solutions of elliptic problems with right-hand side measures, Rendiconti di Matematica 15 (1995), pp. 321-337.

[11] Serrin (J.) .- Pathological solutions of elliptic differential equations, Ann. Scuola Norm. Pisa (1964), pp. 385-387. 